\title{
Dynamic Monitoring of Low Voltage Distribution Network Leakage Detection Device Based on Internet of Things Technology
}

\author{
Yang Wang ${ }^{1, *}$ and Shiqing Wang ${ }^{2}$ \\ ${ }^{1}$ State Grid Liaoning Electric Power Co., LTD., Shenyang, Liaoning 110000, China \\ ${ }^{2}$ Electric Power Research Institute, State Grid Liaoning Electric Power Co., LTD., Shenyang, Liaoning 110000, China
}

\begin{abstract}
The existing dynamic monitoring methods of low-voltage distribution network leakage detection device have been unable to meet the needs of the distribution network in China. Therefore, a dynamic monitoring method of low-voltage distribution network leakage detection device based on Internet of Things technology is proposed. Based on the introduction of the Internet of Things technology sensor sensing technology, the sensor is installed on the leakage detection device to obtain the operation data of the leakage detection device and preprocess it with noise reduction and normalization. At the same time, the statistical analysis of partial discharge signal is carried out to extract the characteristics of fast waveform signal (energy parameters, sample entropy and modal components). Based on the operation data features of the leakage detection device extracted above, the state diagnosis framework of the leakage detection device is built to diagnose the state of the leakage detection device, and the dynamic monitoring of the leakage detection device in low-voltage distribution network is realized. The experimental results show that: compared with the existing methods, the proposed method has stronger anti-interference ability and smaller monitoring error, which fully proves that the proposed method has better application effect.
\end{abstract}

\section{Introduction}

With the rapid growth of China's economy, the continuous increase of power load, the increasing demand of users for power quality, the importance of reactive power compensation in distribution network is also increasing, and the difficulty of reactive power regulation is also increasing. The reasonable distribution of reactive power in power system is the precondition to ensure voltage quality and reduce network loss, and even the material basis to ensure voltage stability of power system. Therefore, reactive power and voltage regulation is one of the core tasks of power system control ${ }^{[1]}$.

Due to the lack of reactive power in the system, when the system is disturbed, the voltage is lower than the critical voltage, resulting in voltage collapse, and the system loses synchronization and collapses. In addition, due to the continuous improvement of the power grid, the increase of urban cable rate and the expansion of load peak valley difference, the situation of excessive reactive power and high voltage in small operation mode is becoming increasingly prominent. The emergence of these two extreme reactive power and voltage imbalances has further aroused the widespread concern of countries all over the world on reactive power optimization ${ }^{22]}$. At present, China is carrying out the interconnection of large-scale regional power grids. The power grid construction is developing towards largescale power grid, ultra-high voltage and long-distance power transmission. The change of transmission power and the switching of high-voltage lines will cause great changes in reactive power. The regulation and control ability of reactive power and grid voltage is required higher and higher, and the difficulty is also increasing. The above situation will lead to leakage of low-voltage distribution network, even minor faults, and even threaten the normal operation of the whole low-voltage distribution network. Therefore, the current low-voltage distribution network will be equipped with leakage detection device to ensure the safety of low-voltage distribution network ${ }^{[3]}$.

It can be seen from the above description that the leakage detection device is the key device in the lowvoltage distribution network. Only by ensuring its stable operation, can the leakage accident of the low-voltage distribution network be effectively avoided. According to the existing research results, the dynamic monitoring method of low-voltage distribution network leakage detection device has been unable to meet the needs of China's distribution network, so this paper proposes a dynamic monitoring method of low-voltage distribution network leakage detection device based on Internet of Things technology. Internet of Things technology originated in the field of media, is the third revolution of information technology industry. Internet of Things refers to the connection of any object with the network through information sensing equipment according to the

*E-mail: yidaiyaorao@163.com 
agreed protocol, and the information exchange and communication of objects through information media, so as to realize intelligent identification, positioning, tracking, supervision and other functions [4]. In short, Internet of Things is the information transmission and control between things, people and things. In the application of Internet of Things, it includes sensor technology, RFID tag, embedded system technology, intelligent technology and nanotechnology. Through the application of Internet of Things technology, we hope to improve the existing problems, and provide more effective guarantee for the stability and safety of lowvoltage distribution network.

\section{Research on dynamic monitoring method of leakage checking device in low voltage distribution network}

\subsection{Introduction of Internet of Things technology}

There are many technologies commonly used in Internet of Things, which can be roughly divided into four categories:

(1)Sensing technology: sensor technology, intelligent sensor technology, barcode technology, RFID technology, product electronic code technology, M2M technology;

(2)Network transmission technology: multi hop network WiFi technology, ZigBee technology, 3G technology, general packet radio service technology, etc;

(3)Remote monitoring technology: global positioning technology, geographic information technology, etc;

(4)Data processing technology: cloud computing technology, information fusion technology, etc.

Among them, RFID, EPC, wireless sensor network, ZigBee, Mobile Internet, M2M, Ethernet, serial communication technology are widely used. According to this research demand, the sensor sensing technology in Internet of Things technology is used to obtain the data of low-voltage distribution network leakage detection device ${ }^{[5]}$.

Wireless sensor network technology, namely WSN, and this technology is the deployment of wireless sensors for wireless network communication, that is to let the monitoring equipment or items "talk". With stable transmission, which can be adjusted at any time and more intelligent ${ }^{[6]}$.

A large number of intelligent sensor nodes with sensing and driving control ability, communication ability and computing ability are deployed in the monitoring area. The nodes can move freely. A fully distributed system without central node and with dynamic topology organization function is formed by wireless communication, which constitutes wireless sensor network. This makes it possible to exchange information between devices, so that managers can grasp the operation status of devices in time ${ }^{[7]}$. It is a new frontier research hotspot with multiple disciplines, which is widely used in power system monitoring, environmental monitoring and other aspects. In wireless sensor networks, sensor nodes are scattered randomly in the monitoring area. With their built-in sensors, nodes can detect the temperature, vibration and other phenomena of devices, and form a network in the form of self-organization. The monitoring data is transmitted to the sink node through multi hop relay mode. The sink node collects the data transmitted by the sensor node and transmits it to the computer for centralized processing. Each sensor node is composed of data acquisition module, data processing and control module, communication module and power supply module, which can be directly embedded into the corresponding equipment and environment, with flexibility and mobility.

\subsection{Data preprocessing of leakage detection device}

The sensor is installed on the leakage detection device, and the relevant data is obtained through the Internet of things sensing technology. In order to make the data truly reflect the operation characteristics of the leakage detection device, it is necessary to preprocess the acquired data to achieve better monitoring effect. The specific preprocessing process is as follows:

In the actual operation process of the leakage detection device, the data of the sensor will inevitably be polluted by noise ${ }^{[8]}$. Although the sensing technology has a certain processing capacity for noise, excessive noise has a destructive effect on the data and the correlation of the data, affecting the monitoring effect of the monitoring method, so it is necessary to de noise the collected data

The common noise reduction method is moving average method, also known as sliding window method. Using this method, the noise reduction is given by the following formula:

$$
x(i)=\sum_{j=i}^{i+1} \frac{x(j)}{l}
$$

In equation (1), $x(i)$ is the denoised value of the $i$ th time data, and $l$ is the length of the sliding window. The average value of samples in the moving window of $i$ time and $i+1$ time is used to replace the measured value of $x(i)$ time.

The types of sensors include current, voltage, power and so on. Different kinds of sensor readings have different dimensions. Therefore, it is necessary to standardize the data to eliminate the adverse effects caused by different dimensions. For the $i$ th measurement parameter $x$, the standardized formula is given by the following formula:

$$
\hat{x}_{i}=\frac{x_{i}-\frac{1}{n} \sum_{j=1}^{n} x_{j}}{\frac{1}{n} \sum_{j=1}^{n} x_{j}^{2}-\left(\frac{1}{n} \sum_{j=1}^{n} x_{j}\right)^{2}}
$$


Through the above process, the data preprocessing of leakage detection device is completed, which lays a solid foundation for the subsequent data analysis and processing.

\subsection{Statistical analysis of partial discharge signal}

Discharge times $n$, apparent discharge quantity $q$ and discharge phase $\varphi$ are the three basic parameters of partial discharge in a power frequency cycle, which constitute the PRPD mode in a single power frequency cycle. But partial discharge has strong randomness, showing strong statistical dispersion of $n, q$ and $\varphi$. Therefore, the general practice is to do statistical processing for $n$ and $q$ in several power frequency cycles, and convert them into one power frequency cycle or unit time, so as to show the statistical regularity, so as to strengthen the PRPD mode and reflect the discharge characteristics of the leakage detection device .

On the basis of $n, q$ and $\varphi$, the following spectra are mainly used at present.

(1) $\varphi-q$ scatter plot: plot the phase and amplitude pairs $\left(\varphi_{i}, q_{i}\right)$ of all discharges in the form of dots in the two-dimensional $\varphi-q$ coordinate system, i.e. $\varphi-q$ scatter plot.

(2) $\varphi-q$ gray scale image: $\varphi-q$ gray scale image is actually the projection of $\varphi-q-n$ threedimensional spectrum image on $\varphi-q$ two-dimensional plane. Firstly, the $\varphi-q$ plane is divided into grids, and the number of grids reflects the resolution of the gray image. Then the discharge frequency $n$ in each grid is counted, and the value of $n$ is converted into gray value according to certain rules. Generally, the minimum and maximum value of $n$ are regarded as the minimum gray level and the maximum gray level respectively. After all the grids are drawn, the $\varphi-q$ gray scale image is obtained.

(3) Two dimensional histogram: according to different processing methods, the maximum discharge phase distribution, average discharge phase distribution, discharge frequency phase distribution and discharge frequency discharge distribution can be obtained, which are respectively recorded as $\varphi-q_{\max }, \varphi-q_{\text {ave }}, \varphi-n$ and $q-n$.

When the above discharge spectra are directly used as the input of the classifier, the dimension is too high, and further feature extraction is needed. Common features include statistical features of two-dimensional histogram, fractal features of scatter plot, moment features of gray-scale image, etc. The extraction process of basic discharge parameters is as follows.

Because the discharge pulse is usually oscillatory and may have multiple local extremum points, the amplitude of a discharge is generally the one with the largest absolute value among multiple local extremum points, and the discharge phase can be converted from the position of the point in the whole signal. Based on this fact, this paper proposes a new method to extract parameters $\varphi-q-n$ by double threshold filtering. The core idea of this method is to find the discharge point by setting two thresholds to double filter all the local extremum points in the whole signal, so as to realize the extraction of $\varphi-q-n$ parameters. The two thresholds are referred to as longitudinal threshold and transverse threshold, which filter the points from the discharge amplitude and discharge interval respectively. The specific implementation steps of the method are given as follows:

(1) The discrete sequence $P_{d}$ of partial discharge signal is input, and the longitudinal threshold $T_{1}$ and transverse threshold $T_{2}$ are set. Among them, $T_{1}$ is used to limit the minimum discharge amplitude, the point with too small amplitude is not considered as an effective discharge, $T_{2}$ is used to limit the minimum discharge interval, and the extreme points in the interval are considered to belong to the same pulse. Their values are determined by experience or repeated experiments according to signal characteristics, and the same values can be used for signals with the same monitoring source .

(2) By scanning $T_{2}$, all local maxima and minima are detected, and the sequence numbers of these extreme points in the original signal sequence are saved in vectors $I_{\max }$ and $I_{\min }$ respectively. Whether a discrete point $\left(x_{i}, y_{i}\right)$ in the signal is a local maximum point is determined by the following formula.

$$
\frac{d y_{i-1}}{d x_{i-1}}>0, \frac{d y_{i+1}}{d x_{i+1}}<0 \text { (3) }
$$

In equation (3), $\frac{d y_{i-1}}{d x_{i-1}}$ and $\frac{d y_{i+1}}{d x_{i+1}}$ represent the differential coefficients before and after point $\left(x_{i}, y_{i}\right)$ respectively.

(3) The absolute values of the amplitude of the extreme points indicated in $I_{\max }$ and $I_{\min }$ are compared with the preset longitudinal threshold $T_{1}$ in turn, and the extreme points whose absolute values are less than $T_{1}$ are eliminated to obtain the updated $I_{\max }$ and $I_{\min }$.

(4) The ordinal values in $I_{\max }$ and $I_{\min }$ are combined into a new vector $I_{\mathrm{m}}$ in ascending order.

(5) Calculate the distance between adjacent extreme points indicated in $I_{\mathrm{m}}$, and save the result in vector $D_{i f f I}$. the calculation method is shown in equation 4.

$$
d(i)=\left\{\begin{array}{cc}
x(i)-0 & i=0 \\
x(i)-x(i-1) & i>0
\end{array}\right.
$$


In equation (4), $d(i)$ refers to the $i$ th value in vector $D_{i f f I}, i$ counts from $0 ; x(i)$ refers to the $i$ th value in vector IM, $i$ counts from 0 .

(6) The distance values in the vector $D_{i f f I}$ are compared with the transverse threshold $T_{2}$ in turn. When the distance value $d(i)<T_{2}$, it means that the current extreme point $x(i)$ and the last extreme point $x(i-1)$ belong to the same pulse, then mark the extreme point with large absolute value. When $d(i) \geq T_{2}$ is found, it means that $x(i)$ and $x(i-1)$ belong to two discharge pulses. At this time, the amplitude and phase of the last discharge pulse are determined immediately according to the marked extreme points, which are stored in vectors $Q_{p_{d}}$ and $\Phi_{p_{d}}$ respectively, and the discharge times $\mathrm{n}$ is accumulated .

(7) The basic parameters of PD signal analyzed at present are output. The total discharge times $N_{p_{d}}$, the amplitude $Q_{p_{d}}$ of each discharge and the corresponding discharge phases $\Phi_{p_{d}}$ and $N_{p_{d}}$ are consistent with the length of vectors $Q_{p_{d}}$ and $\Phi_{p_{d}}$.

\subsection{Fast waveform signal processing}

There is a lot of valuable information in the time series waveform signal of dynamic monitoring of leakage detection device, but it is usually difficult to be directly applied to the status evaluation of the device, so signal analysis algorithm is needed for preprocessing and feature extraction. Fast Fourier transform (FFT) is the most classical analysis method, which can transform the waveform signal from time domain to frequency domain, so as to carry out digital filtering, spectrum analysis, feature extraction and other operations in frequency domain. EMD algorithm decomposes the signal into the sum of several components according to the local characteristics of the signal. However, the EMD algorithm has the inherent frequency mode aliasing problem, and EEMD algorithm uses noise aided technology to solve the problem. This study selects the second method to extract the characteristics of fast waveform signal .

(1) Energy parameters

Due to the time domain or frequency domain characteristic parameters of a single scale are easy to be disturbed by noise, this paper first uses EEMD to carry out adaptive multi-scale decomposition of partial discharge signal, and then constructs the relative energy value of each IMF component as the characteristic quantity of partial discharge type identification. Given a partial discharge signal $x(i), i=0,1,2, \cdots, N-1$. The steps are as follows:

Step 1: EEMD of $x(i)$ to get $n$ components $h_{j}(i)$ including the remainder;

Step 2: calculate the energy value of each component, and the calculation formula is

$$
E(j)=\sum_{i=0}^{N-1} h_{j}(i)^{2}
$$

Step 3: normalize the energy sequence $E(j)$ to get the relative energy value sequence. The formula is

$$
R E(j)=\frac{E(j)}{\sum_{j=1}^{n} E(j)}(6)
$$

(2) Sample entropy

Sample entropy is an improvement of approximate entropy, which is used to measure the complexity of time series. It has small dependence on the length of the series, strong stability and noise resistance, and has a wide range of applications. Therefore, the complexity of PD signals on different scales can be described by the EEMD based SpEn.

Given the modal component $h(i)$, $i=0,1,2, \cdots, N-1$, the calculation process of sample entropy is as follows:

Step 1: given the mode dimension $m$, the mode component $h(i)$ composed of $N$ data points is composed of $m$-dimensional vector;

Step 2: calculate the distance between vectors $Y(i)$ and $Y(j)$ for each $i$ value;

Step 3: given the similarity tolerance $r$, count the number of $i$ values satisfying $d(i, j)<r$, which is called the template statistic number, and record it as num $(d(i, j)<r)$. Calculate the ratio of this number to the total distance $N-m+1$, and record it as $B_{i}^{m}(r)$, and record the average value as $B^{m}(r)$;

Step 4: increase the dimension to $m+1$, repeat steps 1 to 3 to get $B^{m+1}(r)$, then the sample entropy is $\operatorname{SpEn}(m, r)$.

(3) Modal component

EMD algorithm has the characteristics of binary filter banks, and the number of IMF decomposed from signal is generally determined by equation (7).

$$
n_{\text {sifft }}=\left\lfloor\log _{2} N\right\rfloor-1(7)
$$

In equation (7), $N$ is the signal length. When $N=$ $10000, n_{\text {sifft }}=12$. If the relative energy value $R E(j)$ and the sample entropy $\operatorname{SpEn}(m, r)$ are calculated for all the IMF, the worthless non discharge information will 
be introduced, and the dimension of the feature vector obtained is too high, which will have an adverse impact on the subsequent pattern recognition process. Therefore, in this paper, the number of features is determined by calculating the correlation coefficient between each component and the original signal, and the most relevant four IMF are selected to calculate $R E(j)$ and $\operatorname{SpEn}(m, r)$ to form the feature vector .

\subsection{State diagnosis of leakage checking device}

In order to monitor the leakage detection device online, the incremental VPMCD method is deployed on the Storm real-time processing platform, and a dynamic monitoring method of leakage detection device based on Storm and IVPMCD is proposed. The real-time processing framework based on Storm is shown in Figure 1.

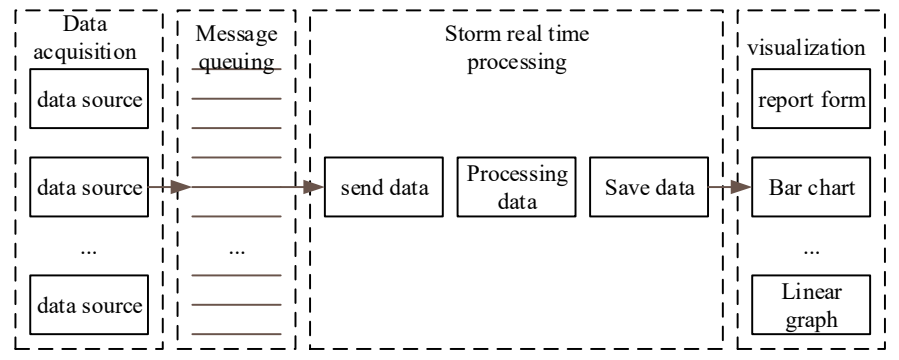

Figure 1 Real time processing framework based on Storm

In this paper, Kafka is introduced into the real-time processing framework to collect the condition monitoring data from a large number of power equipment and distribute them to Storm platform for processing, which plays a role of data collection and buffering between the data source and the processing system. Through the above process, the dynamic monitoring of the low-voltage distribution network leakage checking device can be realized, and the state of the leakage checking device can be diagnosed, so as to provide more effective guarantee for the stable operation of the low-voltage distribution network.

\section{Experiment and result analysis}

In order to verify the performance difference between the proposed method and the existing methods, MATLAB software is used to design simulation experiments. The experimental process is as follows.

\subsection{Selection of subjects}

In this study, the intelligent leakage detection device based on STM32 is selected as the experimental object, and its state safety monitoring indicators are as follows:

The first is power quality. These users have high requirements for voltage, grid frequency, harmonic and other electric energy indicators, and a little deviation will produce a large number of defective products, resulting in serious economic losses. The second is the line load rate. In the low-voltage distribution network of users, when the current passes through the power supply line, the conductor will emit heat. The larger the current is, the more serious the heating of the conductor will be. Long term overload condition will make the insulation of the line aging and increase the possibility of electrical fire. The third is residual current. The residual current is defined as the part where the sum of phase and neutral current vectors is less than zero. The leakage current is generally $\mathrm{mA}$ level, and the over-current protection can not act at this time, so it must be protected by residual current detection. The fourth is short circuit current. If the user line is short circuited frequently, the probability of leakage will be greatly increased.

\subsection{Analysis of experimental results}

Based on the selected experimental objects, the dynamic monitoring experiment of low-voltage distribution network leakage detection device is carried out, and the performance of the method is displayed by the sensor anti-interference and monitoring error. The analysis process of specific experimental results is as follows.

\subsubsection{Analysis of sensor anti-interference}

Under laboratory conditions, the detection performance of the sensor is good, which can basically meet the detection requirements under the premise of less use of electrical appliances in the surrounding environment. But in reality, the electromagnetic environment is more complex than the laboratory environment, so considering the anti-interference of sensor detection. In the laboratory, the load uses a $25 \mathrm{~W}$ bulb. The device detects the magnetic field generated by its current, and at the same time places a mobile phone next to the sensor and is in a conversation. The oscilloscope is used as the display device. The experimental test diagram is shown in Figure 2. 


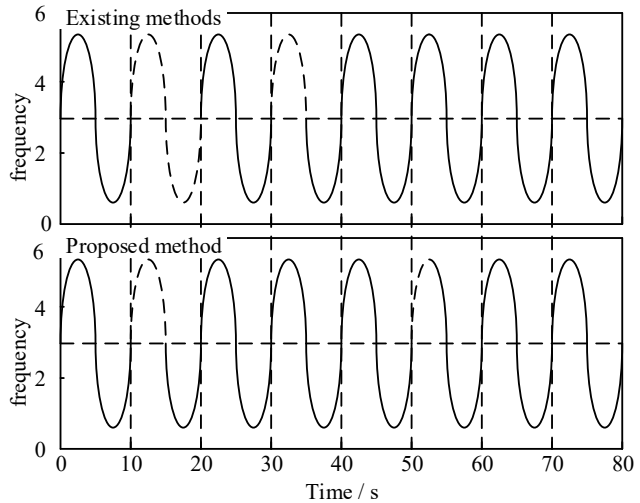

Figure 2 Waveform of monitoring experiment

As shown in Figure 2, compared with the existing methods, the proposed method has fewer missing parts in the waveform, which indicates that the proposed method has stronger anti-interference ability.

\subsubsection{Analysis of monitoring error}

The monitoring error data obtained by experiment is shown in Table 1.

Table 1 Monitoring error data sheet

\begin{tabular}{ccc}
\hline $\begin{array}{c}\text { Number of } \\
\text { experiments }\end{array}$ & $\begin{array}{c}\text { Existing } \\
\text { methods }\end{array}$ & $\begin{array}{c}\text { Proposed } \\
\text { method }\end{array}$ \\
\hline 1 & $26.59 \%$ & $10.01 \%$ \\
2 & $30.12 \%$ & $12.45 \%$ \\
3 & $35.48 \%$ & $11.23 \%$ \\
4 & $33.12 \%$ & $12.40 \%$ \\
5 & $25.10 \%$ & $13.25 \%$ \\
\hline
\end{tabular}

As shown in Table 1, the monitoring error data range of the existing method is $25.10 \%-35.48 \%$, and that of the proposed method is $10.01 \%-13.25 \%$. Through the data comparison, the monitoring error of the proposed method is smaller.

The experimental results show that: compared with the existing methods, the proposed method has stronger anti-interference ability and smaller monitoring error, which fully proves that the proposed method has better application effect.

\section{Conclusion}

This study uses Internet of Things technology to propose a new dynamic monitoring method of low-voltage distribution network leakage detection device, which greatly improves the sensor anti-interference, reduces the monitoring error, provides more effective support for the stable operation of low-voltage distribution network, and also provides a certain reference for the monitoring research of leakage detection device.

\section{References}

1. Antoni, Papi, Blai. Robust and Fast State Estimation for Poorly-Observable Low Voltage Distribution
Networks Based on the Kalman Filter Algorithm[J]. Energies, 2019, 12(23):4457-4457.

2. Qian W, Zhou N, Wu J, et al. Probabilistic ShortCircuit Current in Active Distribution Networks Considering Low Voltage Ride-Through of Photovoltaic Generation[J]. IEEE Access, 2019, 7(99):140071-140083.

3. Qin A, Guo L, You Z, et al. Research on automatic monitoring method of face milling cutter wear based on dynamic image sequence[J]. The International Journal of Advanced Manufacturing Technology, 2020, 110(11-12):1-12.

4. Ray P P, Dash D, De D. Internet of things-based real-time model study on e-healthcare: Device, message service and dew computing $[\mathrm{J}]$. Computer Networks, 2019, 149(11):226-239.

5. Yao M, Zhu Y, Li J, et al. Research on Predicting Line Loss Rate in Low Voltage Distribution Network Based on Gradient Boosting Decision Tree[J]. Energies, 2019, 12(13):2522-2522.

6. Gao S, Tian G, Dai X, et al. A Novel Distributed Linear-Spatial-Array Sensing System Based on Multichannel LPWAN for Large-Scale Blast Wave Monitoring[J]. IEEE Internet of Things Journal, 2019, 6(6):9679-9688.

7. Song L, Sun G, Yu H, et al. FBIA: A Fog-Based Identity Authentication Scheme for Privacy Preservation in Internet of Vehicles[J]. IEEE Transactions on Vehicular Technology, 2020, 69(5):5403-5415.

8. Zeraati M, Golshan M E H, Guerrero J M. Voltage Quality Improvement in Low Voltage Distribution Networks Using Reactive Power Capability of Single-Phase PV Inverters[J]. IEEE Transactions on Smart Grid, 2019, 10(5):5057-5065. 